\title{
Microwave Signal Generation Using Self-Heterodyning of a Fast Wavelength Switching SG-DBR Laser
}

\author{
Michael A. Bernacil, Shane O'Connor, Ben Maher, Andrew Dekelaita, and Dennis Derickson \\ California Polytechnic State University (Cal Poly), 1 Grand Avenue, San Luis Obispo, CA, 93407, USA
}

\begin{abstract}
Microwave signal generation using selfheterodyning of a single wavelength tunable SG-DBR laser is demonstrated. Microwave signals are established by quickly hopping back and forth between two optical wavelengths. These wavelengths are made time coincident through a delay line interferometer. The output of the interferometer is photodetected resulting in a CW microwave signal. This method does not require conventional master-slave laser configurations, external microwave drive sources, or a mode locked laser used to beat dual longitudinal modes. Microwave signals up to $12 \mathrm{GHz}$ have been measured by frequency modulating the phase section of the SG-DBR laser with low frequency RF square wave input currents. Millimeter wave difference frequencies are easily available from the SG-DBR.

Temperature robustness of SG-DBR self-heterodyne signals was also investigated. While each laser wavelength by itself changes by $0.12 \mathrm{~nm} / \mathrm{C}(21 \mathrm{GHz} / \mathrm{C})$, the difference frequency temperature coefficient is well less than $30 \mathrm{MHz} / \mathrm{C}$ typically.

Index Terms - Distributed Bragg reflector (DBR) lasers, distributed feedback (DFB) lasers, microwave generation, microwave oscillators, millimeter wave generation, optical selfheterodyning, semiconductor lasers, wavelength tunable lasers.
\end{abstract}

\section{INTRODUCTION}

Tunable diode lasers are currently the focus of much attention in the telecommunications industry, as they are expected to become essential components for the next generation of dense wavelength division multiplexing systems [1]. Sampled-grating Distributed Bragg Reflector (SG-DBR) single-chip tunable lasers are a recent component used in DWDM systems. These $3 \mathrm{~mm}$ long diode laser chips can tune over the entire 1530 to $1560 \mathrm{~nm}$ telecommunication band with three current inputs controlling the laser wavelength. Previous research into SG-DBR lasers has led to demonstrations of fast wavelength switching capabilities of fewer than 5-ns for a 64channel laser with switching accuracy of $\pm 12 \mathrm{GHz}$ [2]. This paper demonstrates microwave signal generation using this fast wavelength switching feature.

\section{REVIEW OF MICROWAVE SIGNAL GENERATION USING OPTO-ELECTRONICS}

Optically injected semiconductor lasers have recently been used in photonic microwave generation [3]. A common method of generating microwave signals from optical components utilizes a master-slave laser arrangement. In a master-slave configuration with sufficient injection power, the optical frequency of the slave laser is injection locked to that of the master laser. By properly adjusting the injection strength and the frequency detuning, instability occurs through Hopf bifurcation into the period-one dynamical state. The result is a tunable microwave oscillation in the optical power of the slave laser [3].

A second category used to create microwave signals is based on an external modulation technique. The external modulator can either be an electro-optic intensity modulator or a phase modulator. A system that could generate millimeterwave signals using an external intensity modulator was proposed in 1992 by O'Reilly et al. [4]. A frequency-doubled electrical signal was optically generated by biasing the intensity modulator to suppress the optical carrier and the even-order optical sidebands. A $36 \mathrm{GHz}$ millimeter-wave signal was generated when the intensity modulator was driven by an $18 \mathrm{GHz}$ microwave signal. A key advantage of this approach is that frequency-doubled signals can be generated with a relatively low-speed external modulator. However, similar to approaches in the first category, a high quality microwave reference source is required [5].

The third category involves avoiding a reference microwave source, or microwave drive signal. Microwave or millimeter-wave signals are generated by using an individual laser source. To obtain a beat signal at the output of a photodetector, the laser should have either a single wavelength with dual longitudinal modes, i.e. a mode-locked laser, or two wavelengths operating in single longitudinal mode (SLM) for each wavelength. The beating of the dual longitudinal modes or the two SLM wavelengths would generate a microwave signal with the required frequency [5].

A recent attempt to generate microwave signals using DFB-laser wavelength chirp has been explored in [6]. A distributed-feedback laser was used to generate microwave signals using an interferometer to create optical difference frequencies. This was achieved by amplitude modulating the laser bias current and utilizing the frequency-modulated byproduct of the output laser light. An RF triangular and rectangular waveform was injected into the laser bias current section with a modulation period corresponding to an oddinteger multiple of twice the delay period of the interferometer. The DFB chirp created an optical difference frequency used to generate a microwave signal at the output of the interferometer. The optical frequency, or wavelength, difference was incident onto a $0-50 \mathrm{GHz}$ bandwidth photodetector and the generated microwave signals were observed with an electrical spectrum analyzer. 


\section{MICROWAVE SIGNAL GENERATION WITH SG-DBR LASERS}

This work utilizes SG-DBR lasers for photonic microwaves signal generation. These lasers offer many desirable features such as fast switching times $\sim 5 \mathrm{~ns}$, good side mode suppression ratios (Ratios $<37 \mathrm{~dB}$ ), broadwavelength tuning ranges $\sim 40 \mathrm{~nm}(1528-1568 \mathrm{~nm}$ or $1568-$ $1608 \mathrm{~nm}$ ), as well as lasing stability \& repeatability. Furthermore, compared to conventional electronic processing, advantages of photonic processing include ultra high bandwidth, immunity to electromagnetic interference, flexibility, etc., which brings attractive application prospects in microwave and millimeter wave engineering [7].

This research utilizes a self-heterodyne approach to photonic microwave signal generation using fast wavelengthswitching SG-DBR lasers. Our experimental setup does not require wavelength locking of a master to a slave laser, or an external microwave source. Instead microwave signals are generated through a single laser design by self-heterodyning. Signals up to $12 \mathrm{GHz}$ have been obtained, as well as difference-frequency temperature stabilities of less than 30 $\mathrm{MHz} / \mathrm{C}$. In this paper, we report the experimental demonstration of this method.

\section{EXPERIMENT}

Fig. 1 shows a top view of the SG-DBR laser diode chip used in this experiment [1,2]. This laser chip has five segments requiring separate current biases. The gain section provides for the amplification needed to obtain stimulated emission. The back mirror and front mirror form currentcontrolled wavelength dependent reflectivity to tune the wavelength of the laser. The phase section performs fine "current-controlled stretching" of the laser cavity to make fine adjustments in the wavelength of the laser.

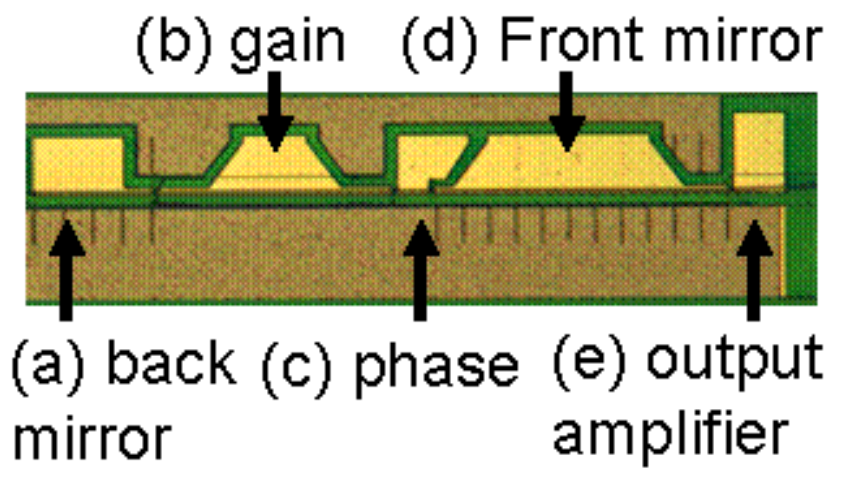

Fig. 1. Top view of the SG-DBR laser utilized in this experiment: The back mirror and front mirrors provide frequency selective reflectivity to select the lasing wavelength. The magnitude of currents driving the front mirror, back mirror, and phase segments control the wavelength of the laser.

Fig. 2 shows the setup to implement single-source photonic microwave signal generation. A low frequency square wave RF signal current is injected into the phase, front mirror, or back mirror segment to modulate the wavelength of the laser. DC current sources provide the required bias current injection into the front \& back mirror facets, gain, phase, and semiconductor optical amplifier (SOA) sections of the laser to establish a nominal operating point.

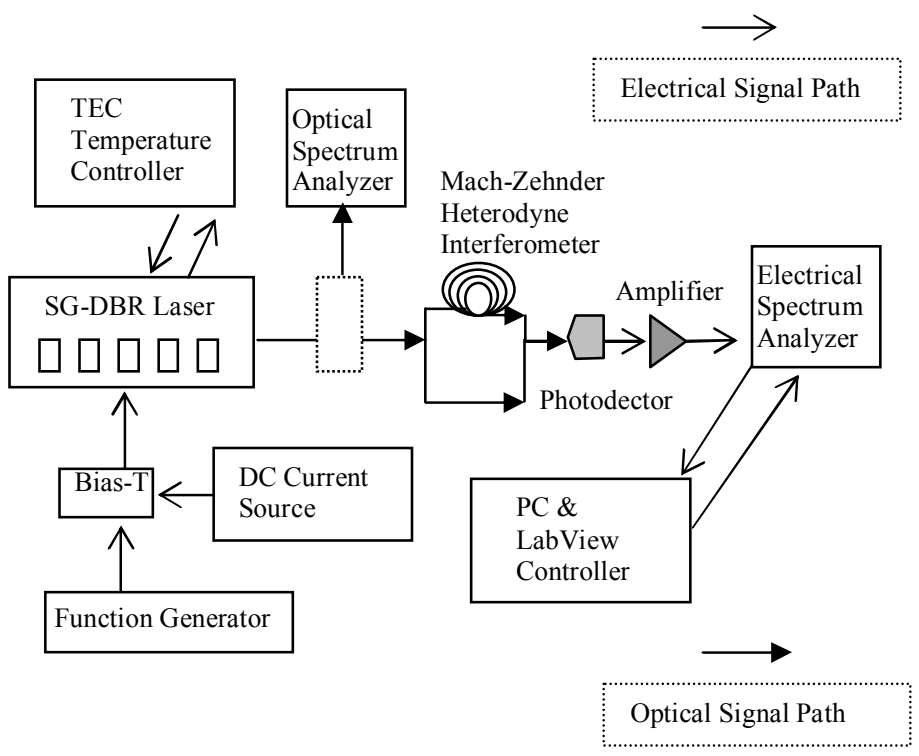

Fig. 2. Experimental setup for obtaining single source photonic microwave signals by self-heterodyne of the SG-DBR output. A low frequency square wave drive from the function generator causes the SG-DBR wavelength to switch between two closely spaced optical frequencies.

The laser chosen for this experiment was the JDSU Inc. MKS-063-B widely tunable laser that was internally modified by the manufacturer to allow for fast wavelength switching. The output of the laser is coupled into a single mode fiber pigtail and sent into a Mach-Zehnder interferometer with a 3.5 $\mu$ s path time delay difference. A square wave drive current with a repetition frequency of $1 /(7 \mu \mathrm{s})$ was applied to the phase section of the SG-DBR laser. The square wave current modulation causes the laser to switch back and forth between two adjacent optical frequencies. The long delay path of the interferometer builds up a 0 to $3.5 \mu$ s time record at wavelength-1. The current drive then forces the laser to wavelength-2 for the $3.5 \mu \mathrm{s}$ to $7 \mu$ s portion of a modulation period. The output of the Mach-Zehnder interferometer therefore has wavelength-1 and wavelength- 2 overlapping in time. The interferometer beats the two switching optical frequencies together and the output of the photodetector/preamplier is a sinusoid at the difference frequency. This process is referred to as self-heterodyning of the laser signal. The drive current can be adjusted so that the two frequencies can have almost any difference frequency of interest. The packaged tunable laser can switch between any two wavelengths in the 1520 to $1560 \mathrm{~nm}$ range within 
approximately $20 \mathrm{~ns}$ to $80 \mathrm{~ns}$. The signal is then amplified and sent into an electrical spectrum analyzer.

\section{MICROWAVE SIGNAL RESULTS}

Fig. 3 shows the self-heterodyne output obtained by driving the phase section with a square wave for various drive levels. The signal peaks represent the electrical difference frequency between the two-switched optical frequencies. Each signal peak corresponds to a particular square wave peak-to-peak modulation current injected into the phase section of the laser. The square wave modulation frequency was set to $143 \mathrm{kHz}$ corresponding to a $3.5 \mu$ s delay from the $700 \mathrm{~m}$ fiber optic delay line in the interferometer. Note this method does not require a microwave frequency reference source or drive signal.

There are several experimental conditions that contribute to the most favorable generation of microwave signals. These conditions significantly narrow the measured linewidth, provide a reduction in phase and relative intensity noise (RIN), and more. They include, but are not limited to, the follow:

a.) It is important to suppress any electrical noise from the five current sources, in particular the sections of the laser responsible for wavelength switching. As a result, a series of $2.5 \mathrm{~Hz},-3 \mathrm{~dB}$ corner frequency passive low pass filters were built and attached to the input of the SG-DBR bias current sections. The filters provide enough high frequency isolation at the DC current inputs to significantly narrow the laser linewidth measured from the generated microwave signals.

b.) The SG-DBR section being modulated needs to have a steady state drive waveform. The high pass, $-3 \mathrm{~dB}$ corner frequency on the bias-T has to be significantly less than the modulation frequency of the square wave. This is required to mitigate droop effects of the waveform amplitude. As a result, a high pass $3.18 \mathrm{kHz},-3 \mathrm{~dB}$ corner frequency bias- $\mathrm{T}$ was designed and built for this application.

c.) Substantial optical isolation is required within the system to prevent any optical reflections back to the laser source. An inline optical isolator was inserted at the output of the laser fiber pigtail to avoid interference reflections from downstream optical fibers.

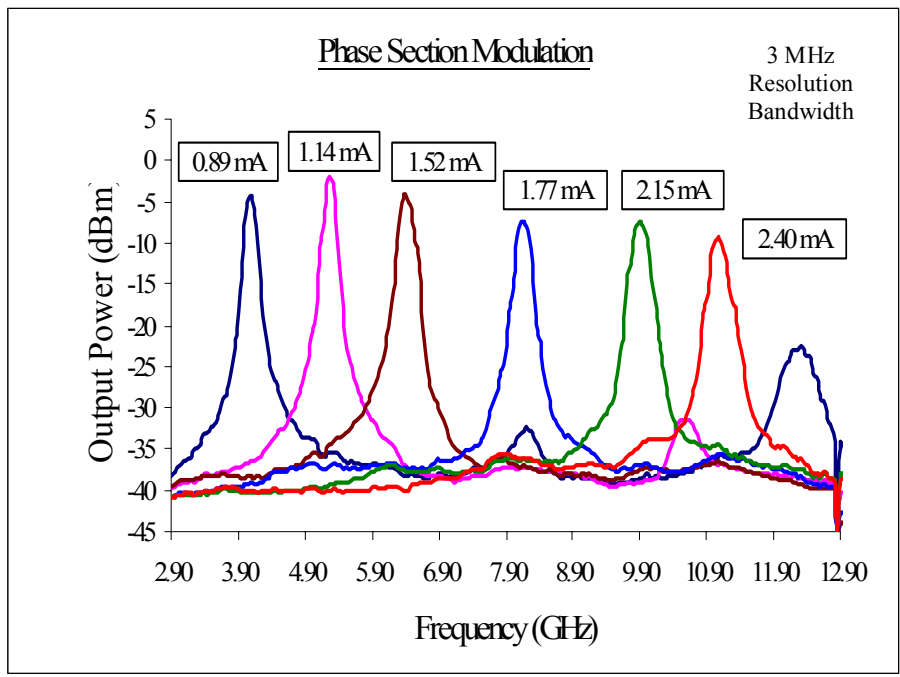

Fig. 3. The output power vs. frequency of generated microwave signals for selected RF modulation inputs. Phase section modulation, $143 \mathrm{kHz}$ carrier frequency, $22^{\circ} \mathrm{C}$ operating temperature.

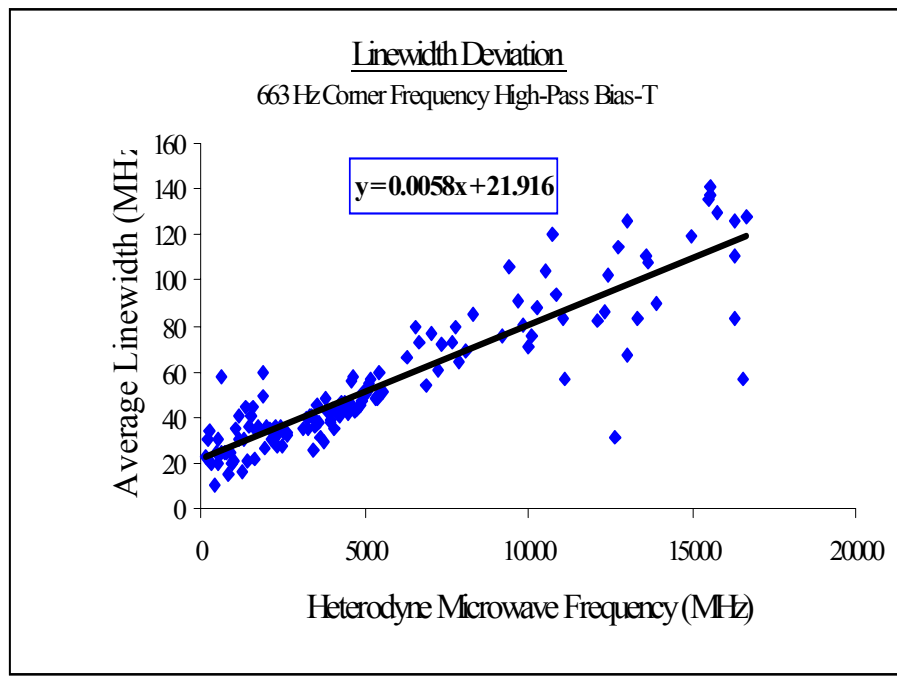

(c)

Fig. 4. Laser linewidth measured from generated microwave signals with respect to heterodyne difference frequency - corresponding to the frequency at the peak of each generated signal.

Fig. 4 shows the change in linewidth with respect to frequency of the generated microwave signals. Notice the 0.0058 slope corresponding to an average change in linewidth in $\mathrm{MHz}$ per generated microwave signal frequency in $\mathrm{MHz}$. 


\section{TEMPERATURE ROBUSTNESS}

This section examines the temperature dependence of the self-heterodyne signal frequency. Fig. 5 illustrates the laser wavelength as a function of temperature for a nominal DC laser bias with front mirror: $20 \mathrm{~mA}$, back mirror: $30 \mathrm{~mA}$, phase section: $5.5 \mathrm{~mA}$, gain section: $100 \mathrm{~mA}$, and SOA: 150 $\mathrm{mA}$. The measured wavelength change was $1.8 \mathrm{~nm}$ for a temperature change of $8^{\circ} \mathrm{C}$. The temperature coefficient was $0.12 \mathrm{~nm} / \mathrm{C}$ or $21 \mathrm{GHz} / \mathrm{C}$.

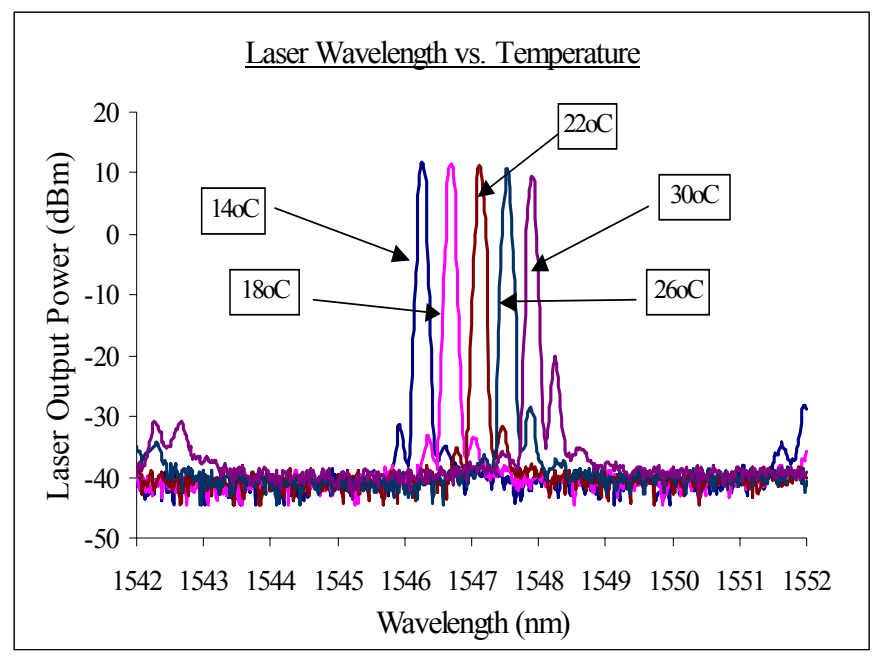

Fig. 5. Wavelength as a function of temperature for a nominal current setting. Note the $0.12 \mathrm{~nm} / \mathrm{C}(21 \mathrm{GHz} / \mathrm{C})$ temperature coefficient of the center wavelength.

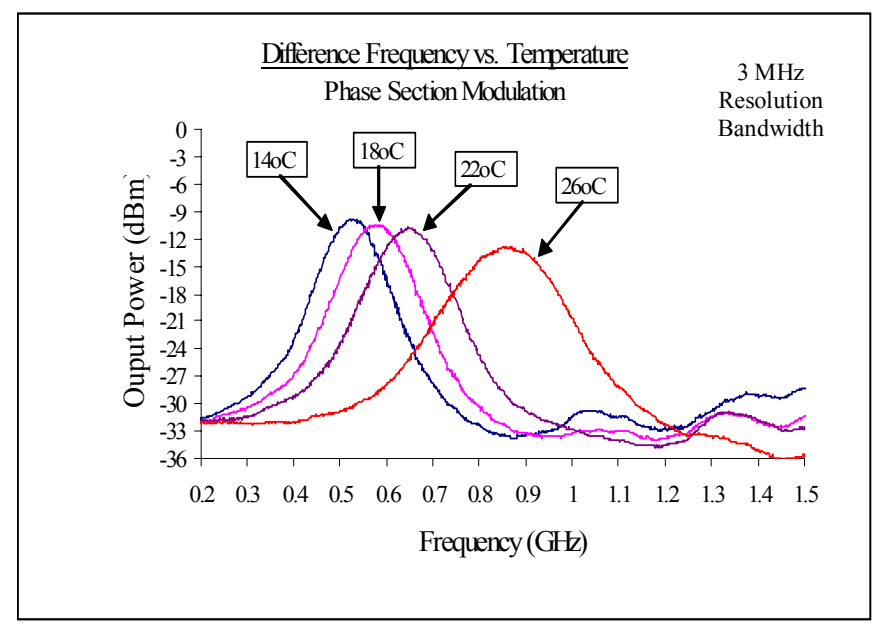

Fig. 6. Self-Heterodyne difference frequency as a function of temperature. The 18 to $22^{\circ} \mathrm{C}$ temperature coefficient of the selfheterodyne difference frequency is $25 \mathrm{MHz} / \mathrm{C}$.

Fig. 6 shows the self-heterodyne difference frequency as a function of temperature for the nominal bias condition of Fig. 5 , and the measurement configuration of Fig. 2. The selfheterodyne temperature coefficient between $18^{\circ} \mathrm{C}$ and $22^{\circ} \mathrm{C}$ was $25 \mathrm{MHz} / \mathrm{C}$.

\section{CONCLUSION}

A technique to generate microwave signals using a single tunable SG-DBR laser in a self-heterodyne configuration is shown. Signal frequencies as high as $12 \mathrm{GHz}$ have been reported in this work (primarily limited by our photodetector). This method of photonic microwave signal generation does not require the use of a master-slave laser configuration, an external microwave drive source, or a mode locked laser.

Furthermore, robustness against temperature has been shown. Additional research into other causes of linewidth broadening involves characterization of thermal induced transients of the laser wavelength through pre-emphasis of the RF square waveform. These investigations will lead to a narrower band closer to the unmodulated $10 \mathrm{MHz}$ linewidth available from this class of diode lasers.

\section{ACKNOWLEDGEMENTS}

The authors would like to thank Susan Portugal for assistance with tunable band pass filters, Michael Biller for supplying low frequency monolithic amplifiers, and Kyle Woolrich for consultation with high \& low frequency amplifier design. This work was sponsored by the Department of Navy, Office of Naval Research, under Award No. N00014-06-1-1111.

\section{REFERENCES}

[1] Gert Sarlet, Geert Morthier, and Roel Baets, "Control of Widely Tunable SSG-DBR Lasers for Dense Wavelength Division Multiplexing," IEEE Trans. Journal of Lightwave Technology, vol. 18, no. 8, pp. 1128-1138, August 2000.

[2] John E. Simsarian, Michael C. Larson, Henry E. Garrett, Hong $\mathrm{Xu}$, and Timothy A. Strand, "Less Than 5-ns Wavelength Switching With an SG-DBR Laser," IEE Photonics Technology Letters, vol. 18, pp. 565-567, February 2003.

[3] Sze-Chun Chan and Jia-Ming Liu, "Frequency Modulation on Single Sideband Using Controlled Dynamics of an Optically Injected Semiconductor Laser," IEE Journal of Quantum Electronics, vol. 42, pp. 699-705, July 2006.

[4] J.J. O'Reilly, P.M. Lane, R. Heidemann, and R. Hofstetter, "Optical generation of very narrow linewidth millimeter wave signals," Electron. Lett., vol. 28, no. 25, pp. 2309-2311, 1992.

[5] Xiangfei Chen, Zhichao Deng, and Jianping Yao, "Photonic Generation of Microwave Signal Using a Dual-Wavelength Single-Longitudinal-Mode Fiber Ring Laser," IEE Trans. Microwave Theory and Techniques, vol. 54, pp. 804-809, Febuary 2006.

[6] Gabor Kovacs, Tibor Berceli, "A Novel Approach to Microwave Signal Generation Utilizing DFB-Laser Wavelength Chirp," $2^{\text {nd }}$ European Microwave Integrated Circuit Conference, pp. 528-531, October 2007, Munich Germany.

[7] Shijun Xiao and Andrew M. Weiner, "Coherent Photonic Processing of Microwave Signals Using Spatial Light Modulators: Programmable Amplitude Filters," IEEE Trans. Journal of Lightwave Technology, vol. 24, pp. 2523-2529, July 2006. 